\title{
INTEROBSERVER VARIATION OF PROSTATIC VOLUME ESTIMATION WITH DIGITAL RECTAL EXAMINATION BY UROLOGICAL STAFFS WITH DIFFERENT EXPERIENCES
}

\author{
WAI C. CHENG, FAI C. NG, KWOK C. CHAN, YUEN H. CHEUNG, WAI L. CHAN, SANG W. \\ WONG
}

\author{
Division of Urology, Department of Surgery, The Chinese University of Hong Kong, Prince of Wales \\ Hospital, Hong Kong, China
}

\begin{abstract}
Purpose: To compare the accuracy of estimating prostatic volume with digital rectal examination (DRE) by urological staffs with different experiences. Measurement of prostatic volume with transrectal ultrasonography (TRUS) serves as the reference standard.

Materials and Methods: Thirty-nine consecutive male patients admitted with acute urinary retention had their prostatic volume estimated with DRE by a urology junior trainee, a urology higher trainee and a trained urologist. All patients had TRUS to measure their prostatic volumes. Pearson correlation coefficients (r) were used to assess the relationships between the prostatic volume measured with TRUS and that estimated with DRE by the 3 urological staffs. Wilcoxon signed ranks tests were used to compare the discrepancies between the prostatic volume measured with TRUS and that estimated with DRE for the 3 Urological staffs, and to assess the inter-observer differences of these discrepancies.

Results: The correlation coefficients for the 3 urological staffs were $r=0.573$ for the urology junior trainee, $r=0.541$ for the urology higher trainee, and $r=0.640$ for the trained urologist. The median discrepancies between the prostatic volume measured with TRUS and that estimated with DRE were $-9.1 \mathrm{~mL}$ for the urology junior trainee, $-1.3 \mathrm{~mL}$ for the urology higher trainee and $0.9 \mathrm{~mL}$ for the trained urologist. These discrepancies were statistically significant only in the case of urology junior trainee $(p=0.015$, Wilcoxon signed ranks test). The difference in these discrepancies was statistically significant only between the urology junior trainee and the trained urologist $(p=0.003$, Wilcoxon signed ranks test).

Conclusions: The trained urologist was more accurate in estimating prostatic volume with DRE than the urology junior trainee.
\end{abstract}

Key words: prostate; physical examination; inter observer variation; ultrasonography Int Braz J Urol. 2004; 30: 466-71

\section{INTRODUCTION}

Estimation of prostatic volume may be useful in a variety of clinical settings. Although minimal correlation had been reported between prostatic volume and lower urinary tract symptoms $(1,2)$, it may help in predicting treatment response and selecting therapeutic options. For example, large prostates may respond better with 5 alpha reductase inhibitors (3). Open prostatectomy rather than transurethral resection of prostate (TURP) should be employed for prostate larger than 80 to $100 \mathrm{~mL}$, though TURP was recently reported to be safe with prostate of 70 to $150 \mathrm{~mL}$ (4). DRE remains the cornerstone of diagnosis of prostate 
cancer. Moreover, the decrease in prostatic volume after hormonal or radiation therapy may be used as an indication of therapeutic efficacy $(5,6)$. Also prostatic volume can help in the interpretation of serum prostate specific antigen levels (7).

Urologists perform numerous DRE everyday. The general belief is that the accuracy of prostatic volume estimation with DRE will improve with experience. The aim of the study is to compare the accuracy of estimating prostatic volume with DRE by urological staffs with different experiences. Measurement of prostatic volume with TRUS serves as the reference standard.

\section{MATERIALS AND METHODS}

Thirty-nine consecutive male patients with acute urinary retention admitted to The Prince of Wales Hospital from July 1998 to September 1998 were included, after signing informed consents.

All patients had their prostatic volume estimated with DRE by a urology junior trainee, a urology higher trainee and a trained urologist, in turn. Each observer had no knowledge of the physical findings of the others. The urology junior trainee had 2 months working experience in urology, whereas the urology higher trainee had 20 months and the trained urologist had over 5 years experience.

All patients had TRUS to measure their prostatic volume. The Bruel \& Kjaer Medical Cheetah Ultrasound Scanner type 2003 was used for all ultrasound examinations, together with the Bruel \& Kjaer Endosonic Multiplane Transducer type 8551 of $7.0 \mathrm{MHz}$, which offered both transverse and sagittal views of the prostate gland. At the area of greatest transverse diameter in the transverse view, the antero-posterior and transverse dimensions of the prostate were measured. Sagittal scanning was then performed and the distance from the base to the apex in the median plane measured the longitudinal dimension. All measurements were made by a single urological staff that had more than 3 years experience in urology, had no knowledge of any of the findings on DRE by his colleagues, and did not perform any DRE on any of the patients prior to their TRUS.
The formula antero-posterior dimension $\mathrm{x}$ transverse dimension $\mathrm{x}$ longitudinal dimension $\mathrm{x} \pi / 6$ was used for the calculation of prostatic volume on TRUS, assuming the prostate gland was elliptical. With this formula, a correlation coefficient of 0.9 had been reported, when compared to actual prostatic weights obtained from prostatectomy or cystoprostatectomy specimens (8).

The Pearson correlation coefficients (r) were employed to assess the relationships between the prostatic volume measured with TRUS and that estimated with DRE for each of the 3 urological staffs.

The discrepancy between the prostatic volume estimated with DRE and that measured with TRUS was calculated for each staff, by subtracting the latter from the former, for each patient. The Wilcoxon signed ranks tests were used to compare these discrepancies for the 3 urological staffs, and to assess the inter-observer differences of these discrepancies for the 3 pairs of urological staffs. It was a non-parametric test of equality of medians for paired groups.

\section{RESULTS}

The studied patients had a mean age of 73.8 years (standard deviation 9.3, range 50 - 89). The mean postvoid residual urine volume was $715 \mathrm{~mL}$ (standard deviation 260, range 400 - 1500).

Scatter plots of measured prostatic volume on TRUS and estimated prostatic volume on DRE for the 3 urological staffs were shown in Figures-1 to 3 . The correlation coefficients for the 3 urological staffs were $r=0.573$ for the urology junior trainee, $r=0.541$ for the urology higher trainee, and $r=0.64$ for the trained urologist. Hence the finger of the trained urologist exhibited the strongest relationship with TRUS.

The median discrepancies between the prostatic volume estimated with DRE and that measured with TRUS were $-9.1 \mathrm{~mL}$ (range -86 to 42.6 ) for the urology junior trainee, $-1.3 \mathrm{~mL}$ (range -96 to 27.3 ) for the urology higher trainee, and $0.9 \mathrm{~mL}$ (range -86 to 29.3 ) for the trained urologist. The urology junior trainee had the largest discrepancy. The urology junior trainee and the urology higher trainee 


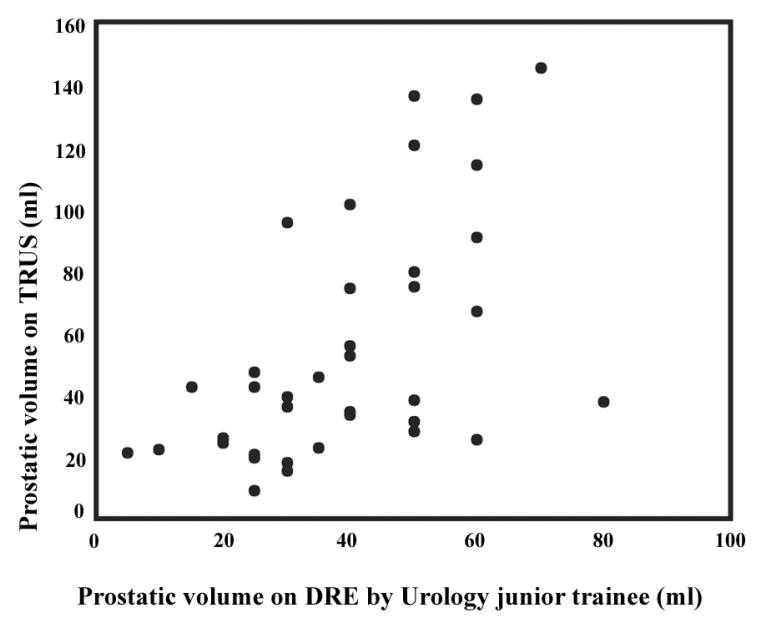

Figure 1 - Scatter plot of transrectal ultrasound (TRUS) volume and digital rectal examination (DRE) volume for urology junior trainee.

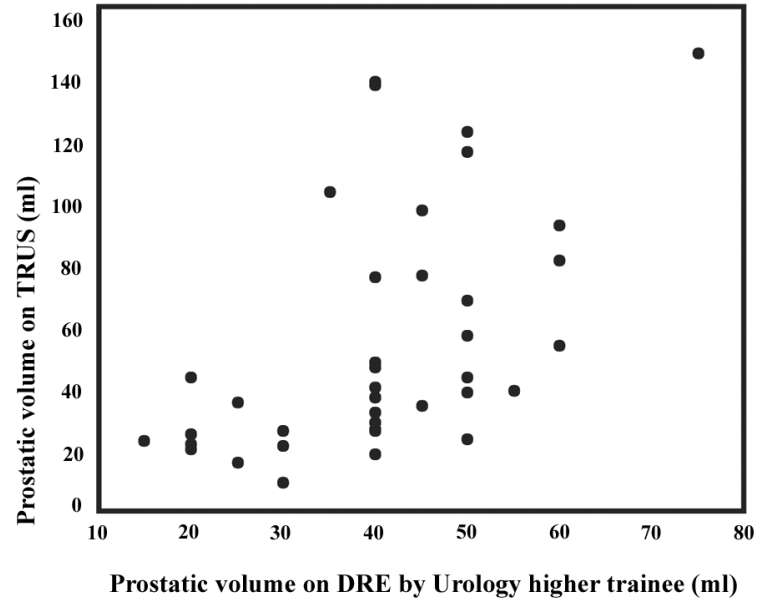

Figure 2-Scatter plot of transrectal ultrasound (TRUS) volume and digital rectal examination (DRE) volume for urology higher trainee.

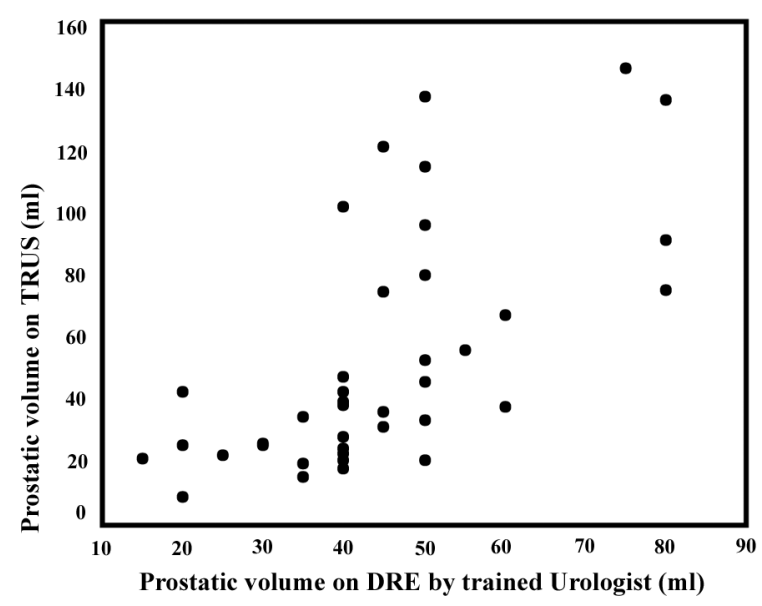

Figure 3 - Scatter plot of transrectal ultrasound (TRUS) volume and digital rectal examination (DRE) volume for trained urologist.

underestimated the prostatic volume in $64 \%$ and $51 \%$ of patients respectively, while the trained urologist overestimated in $54 \%$ of cases. The frequency distributions of discrepancies for the 3 urological staffs were shown in Figures-4 to 6.

The discrepancy was statistically significant for the urology junior trainee $(p=0.015$, Wilcoxon signed ranks test). The discrepancies for the other 2 urological staffs were not statistically significant $(p=0.209$ for the urology higher trainee, and $p=0.610$ for the trained urologist).

There was significant interobserver difference between the discrepancy of the urology junior trainee and that of the trained urologist ( $\mathrm{p}=0.003$, Wilcoxon signed ranks test). The differences in discrepancies for the 2 other pairs of urological staffs (i.e. for the urology junior trainee and the urology higher trainee, and for the urology higher trainee and the trained urologist) were not statistically significant ( $p=0.267$ and 0.096 respectively).

\section{COMMENTS}

DRE of the prostate gland is an important diagnostic tool for the Urologist, in the context of both benign and malignant diseases. Moreover, 


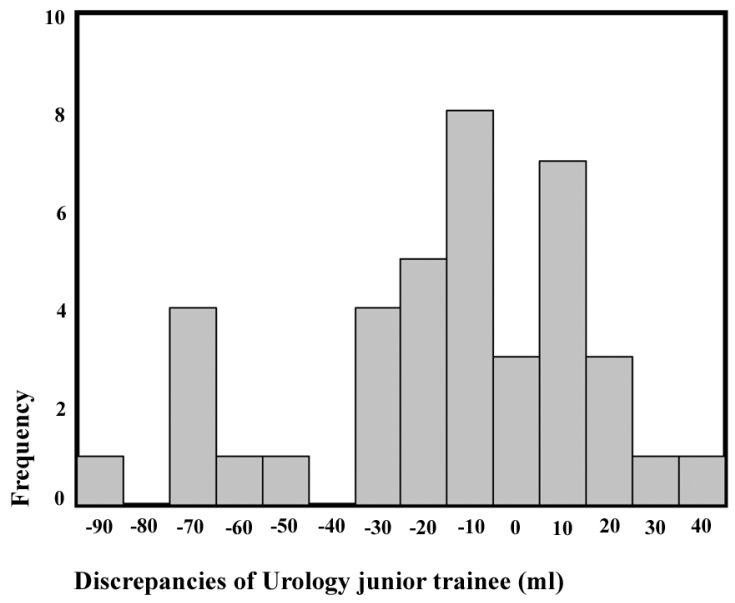

Figure 4 - Frequency distribution of discrepancies for urology junior trainee.

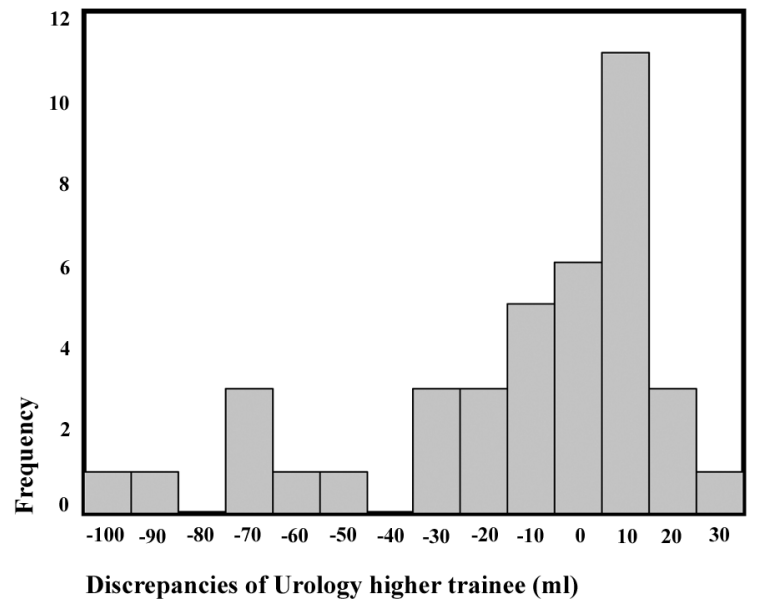

Figure 5 - Frequency distribution of discrepancies for urology higher trainee.

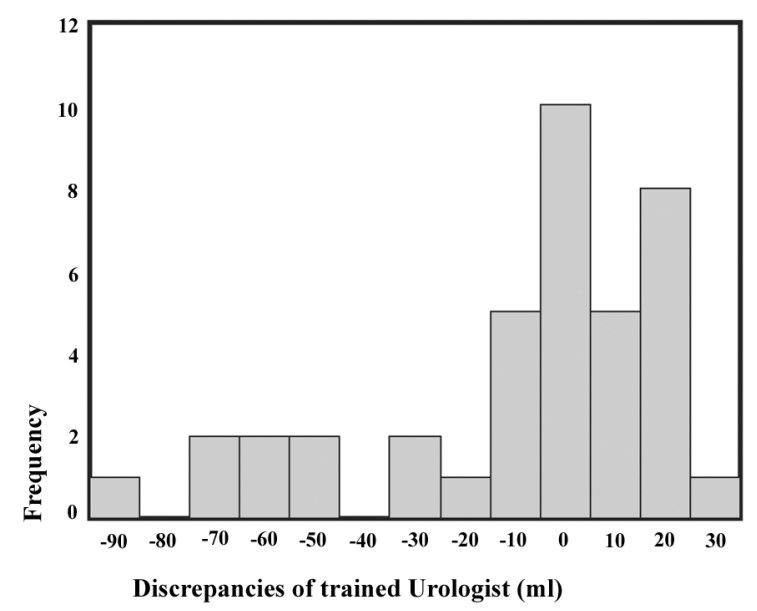

Figure 6 - Frequency distribution of discrepancies for trained urologist.

prostatic volume is a useful parameter in a variety of clinical settings. However, like many other clinical findings, prostatic volume estimated with DRE is subjected to interobserver variation.

Many authors had commented on DRE or prostatic volume. Varenhorst et al. (9) discussed on the interobserver variation in assessment of the prostate with DRE and concluded that DRE had a higher positive predictive value concerning prostate cancer when performed by the urologist rather than the general practitioner. Roehrborn et al. (10) showed significant correlation between prostatic volumes estimated with DRE and that measured with TRUS, and suggested posterior surface area of the prostate correlated with overall volume. Bissada et al. (11) reported that the preoperative estimate of gland weight did not correlate with the actual resected weight during TURP (11). Terris \& Stamey (8) reported on the correlation between prostatic volume measured with TRUS and prostatic specimen weight, using 15 separate methods of volume estimation, and showed that the most accurate method varied according to different volume ranges.

The present study aims at defining the interobserver variation of urological staffs with different experiences in estimating prostatic volume 
with DRE, with measurement with TRUS serving as the reference standard. We choose a group of patients presenting with acute urinary retention, which should not be a selected group, as far as prostatic volume is concerned.

Our patients do have prostates with a wide range of volume on TRUS (9 - $145 \mathrm{~mL})$. Preferably, different formulae should have been used for different volume ranges, according to Terris \& Stamey (8). We employed the widely used elliptical formula (anteroposterior dimension $\mathrm{x}$ transverse dimension $\mathrm{x}$ longitudinal dimension $\mathrm{x} \pi / 6$ ) for the calculation of prostatic volume on TRUS for all patients, as it provides a good correlation with actual prostatic weights (8).

The results of the present study show that both the urology junior trainee and the urology higher trainee have a tendency towards underestimation of prostatic volume, while the trained urologist has a tendency towards overestimation.

The urology junior trainee has the largest discrepancy, which is statistically significant. However even the trained urologist can have a high degree of discrepancy between the prostatic volume estimated with DRE and that measured with TRUS (from -86 to $29.3 \mathrm{~mL}$ ). This observation can be explained by the fact that DRE is assessing only the posterior surface area of a 3 dimensional structure. The intravesical extension of a median lobe and the antero-posterior diameter of the prostate are not taken into account. The antero-posterior diameter can only be estimated by the convexity of the posterior surface of the prostate gland. The consideration of convexity should therefore be included in estimating prostatic volume on DRE.

The high degree of discrepancy between the prostatic volume estimated with DRE and that measured with TRUS of all 3 urological staffs means that DRE only provides a rough estimate of prostatic volume, which is perhaps sufficient to guide therapeutic options, but is inadequate for use in clinical studies involving prostatic volume.

\section{CONCLUSION}

The trained urologist is more accurate in estimating prostatic volume with DRE than a urology junior trainee, as the difference between their discrepancies is statistically significant. The difference between the discrepancies becomes insignificant if the trained urologist and the urology higher trainee are compared. This implies that the technique of DRE can be improved with practice.

\section{REFERENCES}

1. Barry MJ, Cockett AT, Holtgrewe HL, McConnell JD, Sihelnik SA, Winfield HN: Relationship of symptoms of prostatism to commonly used physiological and anatomical measures of the severity of benign prostatic hyperplasia. J Urol. 1993; 150: 351-8.

2. Chute CG, Guess HA, Panser LA, Johnson CL, Jocobsen SJ, Osterling JE, et al.: The non-relationship of urinary symptoms, prostate volume, and uroflow in a population-based sample of men. J Urol. 1993; 149: 356A.

3. Boyle P, Gould AL, Roehrborn CG: Prostate volume predicts outcome of treatment of benign prostatic hyperplasia with finasteride: meta-analysis of randomized clinical trials. Urology. 1996; 48: 398-405.

4. Muzzonigro G, Milanese G, Minardi D, Yehia M, Galosi AB, Dellabella M: Safety and efficacy of transurethral resection of prostate glands up to $150 \mathrm{~mL}$ : a prospective comparative study with 1 year of followup. J Urol. 2004; 172: 611-5.

5. Watanabe H, Ohe H, Ando K, Sawamura Y, Niijima T, Nakamura $S$, et al.: The effect of estramustine phosphate on prostatic cancer estimated by transrectal ultrasonotomography. Prostate. 1981; 2: 155-61.

6. Fujino A, Scardino PT: Transrectal ultrasonography for prostatic cancer: its value in staging and monitoring the response to radiotherapy and chemotherapy. J Urol. 1985; 133: 806-10.

7. Jakobsen H, Torp-Pederson S, Juul N: Ultrasonic evaluation of age-related human prostatic growth and development of benign prostatic hyperplasia. Scand J Urol Nephrol. 1988; 107 (Suppl.): 26-31.

8. Terris MK, Stamey TA: Determination of prostate volume by transrectal ultrasound. J Urol. 1991; 145: 984-7.

9. Varenhorst E, Berglund K, Lofman O, Pedersen K: Inter-observer variation in assessment of the prostate by digital rectal examination. Br J Urol. 1993; 72: 1736.

10. Roehrborn CG, Girman CJ, Rhodes T, Hanson KA, Collins GN, Sech SM, et al.: Correlation between 
prostate size estimated by digital rectal examination and measured by transrectal ultrasound. Urology. 1997; 49: 548-57.
11. Bissada NK, Finkbeiner AE, Redman JF: Accuracy of preoperative estimation of resection weight in transurethral prostatectomy. J Urol. 1976; 116: 201-2.

Received: September 4, 2004

Accepted after revision: October 25, 2004

\author{
$\overline{\text { Correspondence address: }}$ \\ Dr. Cheng Chi Wai \\ Division of Urology, Department of Surgery \\ The Chinese University of Hong Kong \\ Prince of Wales Hospital, Hong Kong, China \\ Fax: + 852 2635-9307 \\ E-mail: drmcheng@ hotmail.com
}

